\title{
Assessment of the association between paraspinal muscle degeneration and quality of life in patients with degenerative lumbar scoliosis
}

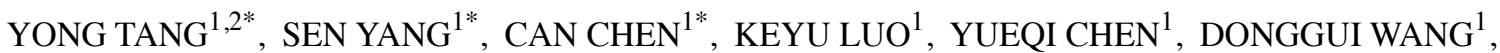 \\ JIULIN TAN ${ }^{1}$, QIJIE DAI ${ }^{1}$, CHENGMIN ZHANG $^{1}$, WENJIE WU ${ }^{1}$, JIANZHONG XU ${ }^{1}$ and FEI LUO ${ }^{1}$ \\ ${ }^{1}$ Department of Orthopaedics, Southwest Hospital, Third Military Medical University, Chongqing 400038; \\ ${ }^{2}$ Department of Orthopaedics, The 72nd Group Army Hospital, Huzhou University, Huzhou, Zhejiang 313000, P.R. China
}

Received November 2, 2019; Accepted March 11, 2020

DOI: $10.3892 /$ etm.2020.8682

\begin{abstract}
The present study aimed to determine the characteristics of multifidus, erector spinae and psoas major degeneration in elderly patients with degenerative lumbar scoliosis (DLS) and the correlation between asymmetric changes and patient quality of life. A total of 49 patients with lumbar scoliosis (DLS group) and 38 healthy individuals (control group) were prospectively examined. The functional cross-sectional area, cross-sectional area difference index (CDI) and fat infiltration rate (FIR) of the multifidus, erector spinae and psoas major at the apical vertebral level were measured using MRI. The visual analogue scale (VAS) score, Oswestry Disability Index (ODI) and 36-item Short Form Health Survey (SF-36) score were used to evaluate patient quality of life. Correlations between the degree of asymmetric muscular degeneration and quality of life were analysed. The CDI of the multifidus, erector spinal and psoas major was higher in the DLS group compared with that in the control group. The CDI of the multifidus was found to be positively associated with the Cobb angle of lumbar scoliosis. Similar results were obtained for fat infiltration between the two groups. In addition, the CDI and FIR difference index of the multifidus was positively correlated with the VAS score and ODI but negatively correlated with the SF-36 score. The quality of life significantly decreased with increasing asymmetric atrophy
\end{abstract}

Correspondence to: Professor Jianzhong Xu or Professor Fei Luo, Department of Orthopaedics, Southwest Hospital, Third Military Medical University, 30 Gaotanyan Street, Shapingba, Chongqing 400038, P.R. China

E-mail: xujzspine@126.com

E-mail: luofeispine@126.com

${ }^{*}$ Contributed equally

Key words: paraspinal muscles, psoas muscles, quality of life, scoliosis and fat infiltration in the multifidus. Thus, strategies to enhance the function of the multifidus may have a positive impact on quality of life (Chinese Clinical Trial Registry, registration date, 2018.11.12; registration no. ChiCTR1800019459.).

\section{Introduction}

Degenerative lumbar scoliosis (DLS) is a degenerative disease in patients $>50$ years with a Cobb angle of $>10^{\circ}$ as the diagnostic criterion (1). Degeneration of the spine may occur along the full length of the spine but is more common in the lumbar spine (2). It is frequently accompanied by a series of degenerative changes involving the vertebrae, intervertebral discs and facet joints (3). Paravertebral muscles (PVMs) and ligaments provide dynamic stability to the lumbar spine (4). Previous studies have focused on the degeneration of intervertebral discs and small joints but did not examine the role of PVMs in supporting spinal balance (5-8). However, the association between PVMs and scoliosis remains to be elucidated.

PVMs are divided into superficial and deep layers. The superficial layer is mainly composed of the erector spinae and the deep layer predominantly consists of the multifidus (9). These are important components of the dynamic spinal stabilization system that provide the power of trunk movements (10). PVM degeneration is characterized by increased fat infiltration and decreased muscle volume (11). PVM degeneration is associated with various lumbar diseases and post-operative complications $(12,13)$. It was reported that the degree of fat infiltration and atrophy of the multifidus is closely linked to the severity of lower back pain (LBP) (14-16). These results suggested that PVM degeneration may be associated with LBP and neurological symptoms. These symptoms have different degrees of impact on the quality of life of DLS patients. However, there is no consensus regarding the association between PVM degeneration and quality of life in DLS.

The aim of the present study was to observe the characteristics of the multifidus, erector spinae and psoas major degeneration in elderly patients with DLS and to study the correlation between asymmetric changes and patient quality of life. 


\section{Materials and methods}

General information. The present prospective clinical study was approved by the Ethics Committee of the Southwest Hospital (First Affiliated Hospital) of the Third Military Medical University (Chongqing, China; approval no. KY201853). All study participants provided written informed consent prior to evaluation and all research activities adhered to the tenets of the Declaration of Helsinki. A total of 49 patients with DLS were recruited at Southwest Hospital, the Third Military Medical University (Chongqing, China) between July 2018 and June 2019 for the DLS group. The inclusion criteria were as follows: Age at onset of DLS (Cobb angle $>10^{\circ}$ ) of $>50$ years; no radiculopathy and no previous therapy for DLS. A total of 38 healthy subjects from outpatient department health check-up were recruited as the control group. All patients were aged $>50$ years, had no degenerative lumbar spine disease and had not received any previous therapy. The exclusion criteria were as follows: Ankylosing spondylitis; spinal tumour; spinal tuberculosis; spinal infection; history of spinal surgery within 2 years; history of traumatic or osteoporotic spinal compression fracture within 1 year; severe pelvic, hip, knee or lower limb lesions; patients with metal implants anywhere in the body, who could not undergo MRI examination; and other serious systemic diseases. Basic characteristics, including sex, age and body mass index (BMI), were not significantly different between the two groups (Table I).

MRI procedure. All patients underwent X-ray and MRI examination using the same equipment. The MRI system was a 3.0 Tesla Imaging System (Siemens Healthineers). T1- and T2-weighted images of the lumbar sagittal plane were captured. The same sequence was used to obtain transverse images at the disc level from T12-L1 to L5-S1. The section thickness was $3 \mathrm{~mm}$, the field of view was $240 \times 240 \mathrm{~mm}$ and each layer was a $512 \times 384$ matrix. The repetition time and echo time was 644 and $9.5 \mathrm{msec}$ for T1-weighted imaging (T1WI) and 4,290 and $93 \mathrm{msec}$ for T2WI, respectively.

MRI evaluation. To minimize the impact of deformity of the spine itself on the morphological measurements of PVMs and psoas, horizontal images in terms of the cross-sectional area (CSA) at the plane of the apex were compared. Images obtained using a T2 sequence were selected for measurement. The region of interest (ROI) was determined by manually tracing the boundaries of the muscles and measured using ImageJ software (v2.1.4.7; National Institutes of Health).

Greyscale images were used to distinguish the pixels of intramural adipose tissue on each disc plane from L1-L2 to L5-S1, with 120 as the threshold (17). The fat infiltration rate (FIR) of the muscles was measured on each side. The FIR difference index (FDI) was measured as the degree of asymmetry between bilateral muscles. The functional CSA (FCSA) was defined as the area of pure muscle without fat using the following formula: FCSA = CSA $\times(1-F I R)$. To compare the FCSA between the concave and convex sides and eliminate the interference of individual differences, the normalized FCSA difference index (CDI) was adopted as the evaluation index (1): $\mathrm{CDI}=\left[1-\left(\mathrm{FCSA}_{\text {concave }} / \mathrm{FCSA}_{\text {conve }}\right)\right] \mathrm{x} 100 \%$. The CDI was also considered as the degree of asymmetric change in the
PVMs. A total of two orthopaedic attending surgeons who had no direct contact with the patients measured all parameters separately. The mean value of their results was used. In a typical case, there was significant asymmetric degeneration in the bilateral paravertebral muscle (Fig. 1).

\section{Quality of life assessment}

Pain. The visual analogue scale (VAS) score was used to evaluate the degree of LBP (18). The VAS score scale ranged from 0 to 10, with a higher VAS score indicating a higher pain intensity.

Dysfunction. The Oswestry Disability Index (ODI) was used to assess dysfunction (19). The ODI scale ranged from 0 to $100 \%$, with a higher ODI indicating more severe dysfunction.

Health-associated quality of life scores. The Medical Outcomes Study 36-item Short Form Health Survey (SF-36) was used to evaluate quality of life in the general population (20). The SF-36 score scale ranged from 0 to $100 \%$, with a higher SF-36 score indicating a better physical condition.

Statistical analysis. Data were analysed using SPSS 17.0 (SPSS, Inc.). Age, VAS score, ODI and SF-36 score were compared using an independent-samples t-test. The CDI and FDI were compared using a Wilcoxon signed-rank test. The FIR was compared using a paired-samples t-test. Differences in sex were analysed using a $\chi^{2}$ test. Pearson correlation analysis was performed to assess the correlation between CDI or FDI and the VAS score, ODI or SF-36 score. P $<0.05$ was considered to indicate a statistically significant difference.

\section{Results}

General information. In the DLS group, 49 patients (sex, 23 males and 26 females; mean age, $61.3 \pm 5.2$ years; age range, 52-83 years; Table I). Among them, 25 and 24 patients exhibited a curved vertex on the left and on the right, respectively. Spinal segments L2-L3 and L3-L4 were the most common apical levels (Fig. 2). The mean Cobb angle was $18.85 \pm 9.22^{\circ}$ (range, $\left.10.7-37.2^{\circ}\right)$. In the Control group, 38 patients were enrolled (sex, 16 males and 22 females; mean age, $62.4 \pm 5.7$ years; age range, 51-82 years; Table I).

Differences between the two groups. The CDI of the multifidus, erector spinae and psoas major were significantly higher in the DLS group compared with those in the control group (Fig. 3). There were no significant correlations between CDI and age, sex, BMI, the side or level of the apex (Table II). However, a association was identified between the CDI of the multifidus at the apical vertebral level and the Cobb angle of lumbar scoliosis ( $\mathrm{P}=0.028$; Table II). It was indicated that the volume of the multifidus was closely associated with the degree of lumbar scoliosis (21). The FIR of the multifidus was also significantly higher on the concave side compared with that on the convex side at the apical vertebral level (1.48 fold-change vs. the convex side), but this difference was not statistically significant for the erector spinae or psoas major (Fig. 4). In addition, the FDI for the multifidus from L1-L5 in the DLS group was significantly higher compared with that in the control group (25.5 fold-change vs. control; Fig. 5). No 
Table I. General characteristics of the study subjects.

\begin{tabular}{lcccc}
\hline Characteristics & DLS group $(\mathrm{n}=49)$ & Control group $(\mathrm{n}=38)$ & \multicolumn{1}{c}{ Statistic } & P-value \\
\hline Sex (male/female) & $23 / 26$ & $16 / 22$ & $\chi^{2}=0.2022$ & 0.6530 \\
Age $($ years) & $61.3 \pm 5.2$ & $62.4 \pm 5.7$ & $\mathrm{t}=0.9383$ & 0.3507 \\
BMI $\left(\mathrm{kg} / \mathrm{m}^{2}\right)$ & $23.3 \pm 2.6$ & $23.7 \pm 2.9$ & $\mathrm{t}=0.6767$ & 0.5004 \\
Cobb angle $\left({ }^{\circ}\right)$ & $18.85 \pm 9.22$ & $2.67 \pm 5.23$ & $\mathrm{t}^{\prime}(0.05)=2.0110$ & 0.0235
\end{tabular}

DLS, degenerative lumbar scoliosis; BMI, body mass index.

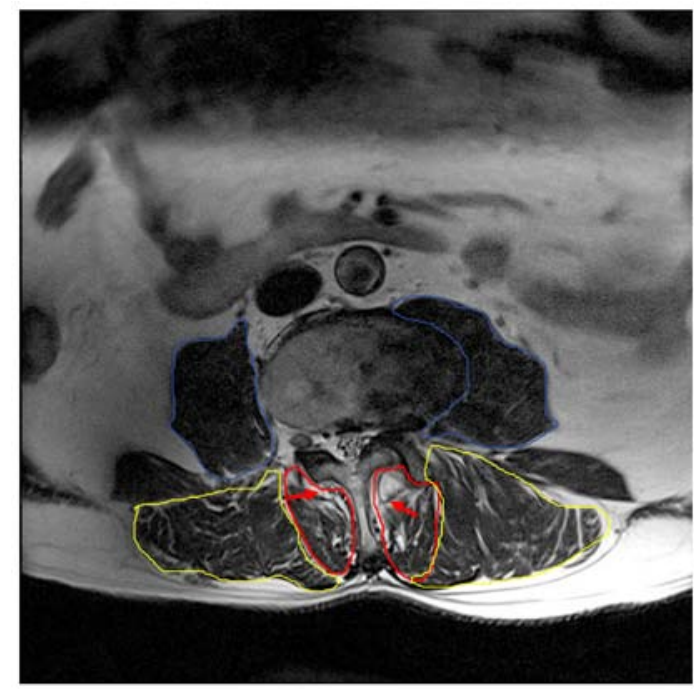

Figure 1. Representative MRI image of a male patient aged 70 years with degenerative lumbar scoliosis. The Cobb angle was $13.8^{\circ}$ and the apical level was L3-L4. The cross-sectional area difference indices of the multifidus, erector spinae and psoas major was $19.6,5.4$ and $15.4 \%$, respectively. The FIR of the multifidus, erector spinae and psoas major were 14.1, 7.4 and $0.3 \%$ on the concave side (left side) and $9.8,6.8$ and $0.1 \%$ on the convex side (right side). The degree of FIR asymmetry was $4.3 \%$. The image indicates asymmetric degeneration of the cross-sectional area and the degree of fat infiltration of the bilateral paraspinal muscles. The red region of interest is the shape of the multifidus. The red arrows indicate a large amount of fat infiltrating the muscle. FIR, fat infiltration rate. The yellow region of interest is the shape of the erector spinae. The blue region of interest is the shape of the psoas major.

statistically significant differences were observed not for that of the erector spinae or psoas major. These results suggested that there was an asymmetrical degree of fat infiltration in the bilateral multifidus in patients with lumbar scoliosis. There was no significant difference in paravertebral muscle of cross-sectional area and fat infiltration in the control group. The contrast between the degeneration and control groups is reflected in the asymmetry between the two sides.

Analysis of associated factors with PVMs change. Comparing the quality of life between the two groups, the VAS score and ODI were also significantly higher in the DLS group (5.48 and 14.39 fold-change vs. control, respectively), while the SF-36 score was significantly lower in the DLS group compared with the control group (66.7\%; Fig. 6). According to the correlation analysis, the CDI of the multifidus at the apical vertebral level was positively correlated with the VAS score as well as with

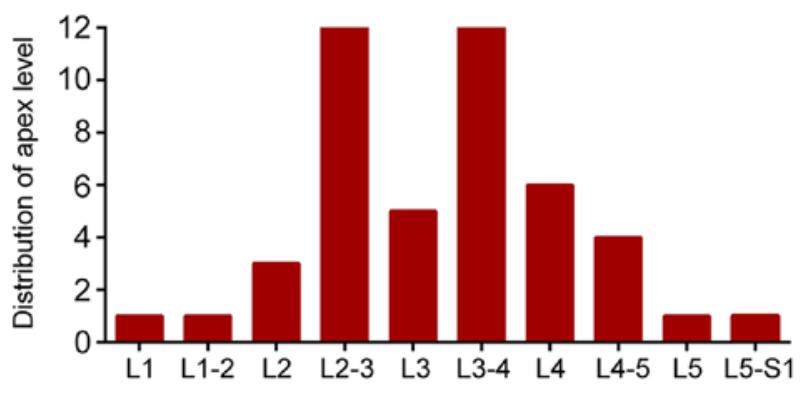

Figure 2. Apex distribution. According to the X-ray image, the apex vertebra is the vertebral body with the longest distance from CSVL, the most horizontal, the greatest degree of rotation and the most obvious wedge-shaped change in the entire bending range. In patients with degenerative lumbar scoliosis, the apical levels of bending were mainly distributed in L2-L3 and L3-L4.
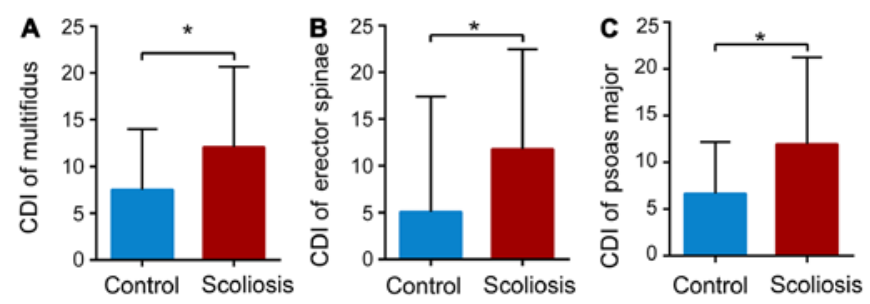

Figure 3. Differences in the CDI of the multifidus, erector spinae and psoas major at the apical level between the degenerative lumbar scoliosis and control groups. CDI of the (A) multifidus, (B) erector spinae and (C) psoas major. ${ }^{*} \mathrm{P}<0.05$. CDI, cross-sectional area difference index.

the ODI but negatively correlated with the SF-36 score in the DLS group (Table III). The FDI of the multifidus from L1-L5 was also positively correlated with the VAS score and ODI and negatively correlated with the SF-36 score. This indicated that the volume and fat infiltration of the multifidus reflected the degree of muscle degeneration. It also indicated that the function of the multifidus was closely associated with the quality of life of patients.

\section{Discussion}

The incidence of DLS increases with age and gradually becomes a public health concern in the elderly population (22). PVMs have an important role in maintaining the stability of the spine. Degeneration of PVMs is associated with various lumbar diseases and post-operative complications $(23,24)$. However, the pathogenesis of DLS remains to be elucidated. 
Table II. Analysis of correlation between the cross-sectional area difference indices of paravertebral and psoas muscles at the apex level with clinicopathological features.

\begin{tabular}{lccc}
\hline & \multicolumn{3}{c}{$\begin{array}{c}\text { Cross-sectional area } \\
\text { difference indices }\end{array}$} \\
\cline { 2 - 4 } $\begin{array}{l}\text { Scoring } \\
\text { system/index }\end{array}$ & Multifidus & $\begin{array}{c}\text { Erector } \\
\text { spinae }\end{array}$ & $\begin{array}{c}\text { Psoas } \\
\text { major }\end{array}$ \\
\hline Sex & & & \\
Coefficient & 0.124 & 0.079 & 0.087 \\
P-value & 0.465 & 0.523 & 0.498 \\
Age & & & \\
Coefficient & 0.189 & 0.113 & 0.065 \\
P-value & 0.381 & 0.485 & 0.573 \\
BMI & & & \\
Coefficient & 0.116 & 0.114 & 0.111 \\
P-value & 0.434 & 0.452 & 0.478 \\
Side of apex & & & \\
Coefficient & 0.042 & 0.056 & 0.078 \\
P-value & 0.706 & 0.672 & 0.598 \\
Level of apex & & & \\
Coefficient & 0.139 & 0.114 & 0.141 \\
P-value & 0.342 & 0.457 & 0.323 \\
Cobb's angle & & & 0.265 \\
Coefficient & 0.325 & 0.224 & 0.095 \\
P-value & 0.028 & 0.123 & \\
\hline
\end{tabular}

BMI, body mass index.
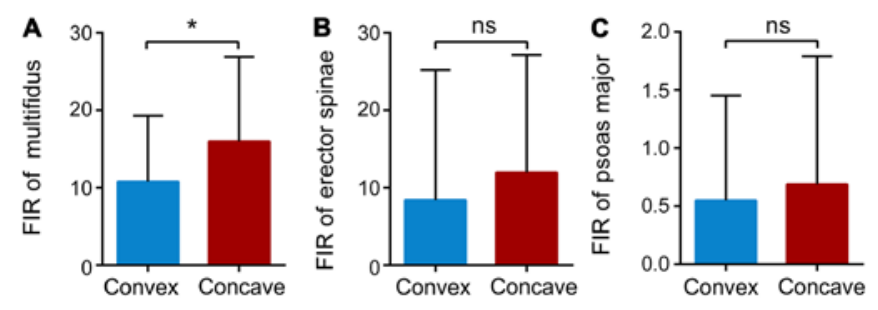

Figure 4. Differences in the FIR of the paravertebral and psoas muscles at the apical level between the concave and convex sides. FIR of the (A) multifidus, (B) erector spinae and (C) psoas major. ${ }^{*} \mathrm{P}<0.05$. ns, no significance; FIR, fat infiltration rate.

The factors that affect the development of DLS are complex and include the vertebral bodies, intervertebral discs, joints, ligaments and muscles (25). Different degrees of PVM degeneration may lead to an imbalance in bilateral lumbar force and aggravate the progression of lumbar scoliosis (26). Current research on lumbar PVMs mainly focuses on the association with the sagittal balance of the spine (26). However, there have been few reports on the association between PVM degeneration and quality of life. Therefore, the present study focused on asymmetric changes in the PVMs and psoas major in patients with degenerative scoliosis and validated the association between PVM degeneration and quality of life.
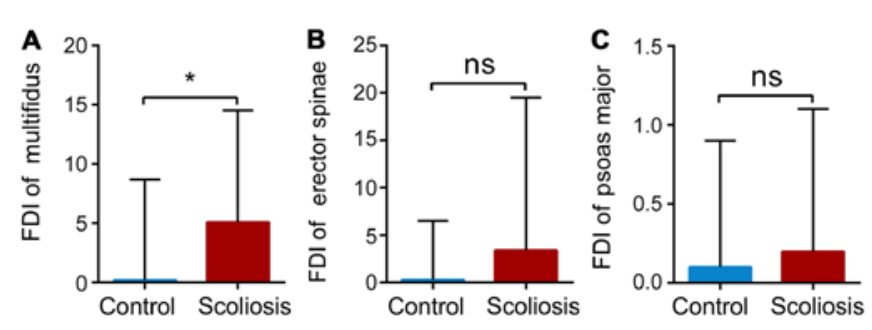

Figure 5. FDI of the paravertebral and psoas muscles at the apical level between the degenerative lumbar scoliosis and control groups. FDI of the (A) multifidus, (B) erector spinae and (C) psoas major. ${ }^{*} \mathrm{P}<0.05$. ns, no significance; FDI, fat infiltration rate difference index.
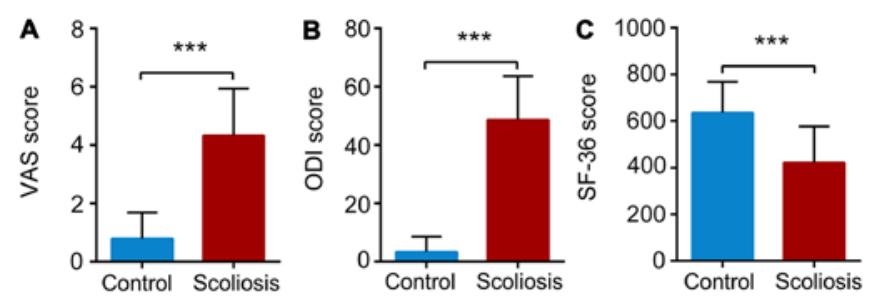

Figure 6. Comparison of quality of life. (A) VAS, (B) ODI and (C) SF-36 scores compared between the degenerative lumbar scoliosis and control groups. ${ }^{* * *} \mathrm{P}<0.001$. VAS, visual analogue scale; ODI, Oswestry Disability Index; SF-36, 36-item Short Form Health Survey.

A decrease in the CSA indicates muscle atrophy and an increase in the FIR reflects muscle degeneration (11). It has been reported that the CSA of the PVMs significantly decreased, while the FIR of the PVMs increased in patients with LBP $(27,28)$. Muscle atrophy and fat infiltration occur simultaneously, which is due to the tendency of myosatellite cells to undergo adipocytic differentiation under pathological conditions (29). Therefore, the degree of PVM change measured by MRI mainly includes changes in the CSA and FIR $(30,31)$. To measure the CSA, the ROI must be drawn along the muscle boundary. Although this method involves a certain amount of subjectivity, studies have proven that it has good internal and external reliability (32). A previous study measured the total muscle CSA, including fat, while others have measured the pure muscle area without adjacent degenerative fat, referred to as the FCSA (33). The difference in bilateral back muscle mass was indicated to be most significant at the apical vertebral level (34). To reduce individual differences, the FCSA on both sides of the apical vertebral level was adopted and the concave side was compared with the convex side to obtain the relative asymmetry. At present, there are no particularly accurate methods for measuring fat infiltration, while MRI is the most accurate method. However, there are numerous methods based on MRI, including fat three-dimensional reconstruction, magnetic resonance spectroscopy and Dixon technology $(35,36)$. Muscular atrophy and fatty infiltration occur simultaneously and there is maximum tension between two sides of the apex level (25). The apex level was selected to eliminate individual and segmental differences for measuring CDI. The infiltration of fat is in the whole muscle. However, when evaluating the degree of fat infiltration, the average muscle fat infiltration rate of all segments of L1 to L5 was selected. The present results indicated that the CDI was only 
Table III. Correlation analysis between the CDI or FDI with VAS, ODI and SF-36 scores.

\begin{tabular}{|c|c|c|c|c|c|c|}
\hline \multirow[b]{2}{*}{ Scoring system/index } & \multicolumn{3}{|c|}{ CDI } & \multicolumn{3}{|c|}{ FDI } \\
\hline & Multifidus & Erector spinae & Psoas major & Multifidus & Erector spinae & Psoas major \\
\hline \multicolumn{7}{|l|}{ VAS } \\
\hline Coefficient & 0.459 & 0.177 & 0.152 & 0.387 & 0.071 & -0.142 \\
\hline P-value & 0.010 & 0.218 & 0.293 & 0.006 & 0.624 & 0.324 \\
\hline \multicolumn{7}{|l|}{ ODI } \\
\hline Coefficient & 0.643 & 0.240 & 0.258 & 0.354 & 0.040 & -0.037 \\
\hline P-value & $<0.001$ & 0.093 & 0.070 & 0.013 & 0.783 & 0.799 \\
\hline \multicolumn{7}{|l|}{ SF-36 } \\
\hline Coefficient & -0.318 & 0.036 & -0.079 & -0.338 & -0.178 & -0.062 \\
\hline $\mathrm{P}$-value & 0.024 & 0.806 & 0.584 & 0.018 & 0.215 & 0.671 \\
\hline
\end{tabular}

CDI, cross-sectional area difference index; FDI, fat infiltration rate difference index; VAS, visual analogue scale; ODI, Oswestry Disability Index; SF-36, 36-item Short Form Health Survey.

positively correlated with the Cobb angle and not correlated with age, sex or BMI.

In the DLS group, there were significant differences in the CSA of the bilateral multifidus, erector spinae and psoas major. Unlike healthy individuals, patients with scoliosis experience asymmetrical pressure loads on both sides of the spine, which may be responsible for asymmetric paraspinal atrophy and fat infiltration (25). On the convex side, due to continuous tensile stress, PVMs are in a state of long-term hyperproliferation, which inhibits adipocytic differentiation (37). The PVMs and psoas major maintain the dynamic stability of the spine (34). The increase in the CSA on the convex side may be the result of balance compensation, which eventually achieves the effect of increasing tensile traction and preventing the spine from leaning further to the concave side (4). The characteristics of fat distribution in the bilateral paraspinal muscles also support this explanation, as muscular atrophy is closely associated with increased fat infiltration (38). Therefore, biomechanical changes may lead to asymmetric changes in the PVMs, which is consistent with earlier research on scoliosis (25).

The strength of muscles is proportional to the CSA, and the CSA of the PVMs is frequently used to represent muscle strength in the lower back (39). Therefore, the CDI of the PVMs may directly reflect the difference in muscle strength between the two sides on the same plane. The present results suggest that the CDI of the multifidus at the apical vertebral level was positively correlated with the Cobb angle. A greater Cobb angle of scoliosis is indicative of a higher asymmetry of the gravity load on the two sides. Biomechanical studies have suggested that the load of the multifidus increases by $1.5 \mathrm{~kg} / \mathrm{cm}^{2}$ for each $1 \mathrm{~cm}$ of deviation from the midline of the vertebral apex on the convex side (40). When the spine is in a neutral position, the multifidus contributes up to $60 \%$ of segment stability (41). The present results indicated that the apical vertebrae of patients with DLS tend to appear in the L2-L3 and L3-L4 segments. The reason may be that the multifidus has a key role in maintaining stability between L2 and L4, which is consistent with a previous report (25). Compared with other lumbar muscles, the multifidus is characterized by short fibres and a large CSA (42). This morphological feature allows the multifidus to exert a large force in a relatively narrow space, controlling the stability of the back of the spine (4). In addition, muscle atrophy on the concave side may be closely linked to increased fat infiltration (38). The FIR exhibited marked inter-individual differences and was closely associated with vertebral segment, sex, age and BMI. The present results indicated that only the FIR of the multifidus on the concave side was significantly higher compared with that on the opposite side at the apical vertebral level. It is possible that, as the multifidus is a medial PVM, it is sensitive to changes in the tensile load (30).

The present study indicated that the CDI and FDI of the multifidus were positively correlated with the VAS score and ODI but negatively correlated with the SF-36 score. PVM degeneration is closely associated with LBP. The majority of patients present with back pain as the primary symptom, which has an adverse impact on quality of life and the capacity of patients to work or study (33). LBP causes patients to further reduce functional exercise of the lower back muscles (43). Large amounts of lactic acid and various metabolites accumulate, resulting in muscle oedema and stimulating or aggravating nerve pain (32). The multifidus gradually becomes undernourished and replaced with adipose tissue, which leads to muscle atrophy (44). Patients with lumbar scoliosis have a higher level of dysfunction compared with the general population, which limits their quality of daily life, and certain patients lose the ability to take care of themselves (45). One study reported that the CSA of the PVMs in patients with chronic LBP was smaller compared with that in the controls (42). Decreased PVM CSA is a risk factor for post-operative pedicle screw loosening (46). One study reported the correlation between pre-operative PVM degeneration and cage displacement after fusion (47). Reduced PVM strength and an increased pre-operative FIA may lead to proximal junctional kyphosis (48). Altogether, asymmetric degeneration of the multifidus reduces the quality of life and may even affect the outcome of surgery. 
The present study has certain shortcomings that require to be mentioned. First, the population of the present study consisted of subjects from only a single centre and the sample size was relatively small. Furthermore, analyses were not performed according to the severity of lumbar scoliosis. In addition, there may have been certain bias. For instance, degenerative disc and endplate degeneration were present in the degenerative group, but not in the control group. These factors were not considered, which may have an impact on the assessment of quality of life. Therefore, it is required to further expand the sample size to eliminate bias in future research. It will be endeavoured to perform detailed studies with large samples through multicentre recruitment.

To conclude, in the present study, patients with DLS had asymmetric degeneration of the PVMs and psoas major. The CDI and FDI of the multifidus are closely correlated with the quality of life. These results may provide a strategy of clinical treatments to improve PVM function in order to improve the quality of life and reduce pain.

\section{Acknowledgements}

Not applicable.

\section{Funding}

The current study was supported by the Major Military Logistics Research Projects (grant no. AWS17J004), The Clinical Medical Research Program of Army Medical University (grant no. 2018XLC3010) and Chongqing graduate research and innovation project (grant no. CYS19369).

\section{Availability of data and materials}

The datasets used and/or analyzed during the present study are available from the corresponding author on reasonable request.

\section{Authors' contributions}

YT, SY, CC designed the study and optimized the experimental scheme, collected clinical samples and wrote papers. KL, YC, DW, JT, QD, CZ and WW performed the experiments and statistical analyses. JX and FL designed the present study and revised the manuscript. All authors read and approved the final manuscript.

\section{Ethics approval and consent to participate}

Ethical approval for this study was obtained from the Ethics Committee of the First Affiliated Hospital, Third Military Medical University (approval no. KY201853). Written informed consent was obtained from all participants prior to the study.

\section{Patient consent for publication}

Not applicable.

\section{Competing interests}

The authors declare that they have no competing interests.

\section{References}

1. Kim H,Lee CK, Yeom JS, et al: Asymmetry of the cross-sectional area of paravertebral and psoas muscle in patients with degenerative scoliosis. Eur Spine J 22: 1332-1338, 2013.

2. Buell TJ, Chen CJ, Nguyen JH, Christiansen PA, Murthy SG, Buchholz AL, Yen CP, Shaffrey ME, Shaffrey CI and Smith JS: Surgical correction of severe adult lumbar scoliosis (major curves $\geq 75^{\circ}$ ): Retrospective analysis with minimum 2-year follow-up. J Neurosurg Spine 31: 1-14, 2019.

3. Voirin-Hertz M, Carvajal Alegria G, Garrigues F, et al: Associations of lumbar scoliosis with presentation of suspected early axial spondyloarthritis. Semin Arthritis Rheum 50: 48-53, 2020.

4. Diebo BG, Shah NV, Boachie-Adjei O, Zhu F, Rothenfluh DA, Paulino CB, Schwab FJ and Lafage V: Adult spinal deformity. Lancet 394: 160-172, 2019.

5. Yaltırık K, Güdü BO, Işık Y, Altunok Ç, Tipi U and Atalay B: Volumetric Muscle Measurements Indicate Significant Muscle Degeneration in Single-Level Disc Herniation Patients. World Neurosurg 116: e500-e504, 2018.

6. Doktor K, Jensen TS, Christensen HW, Fredberg U, Kindt M, Boyle E and Hartvigsen J: Degenerative findings in lumbar spine MRI: An inter-rater reliability study involving three raters. Chiropr Man Therap 28: 8, 2020.

7. Haddas R, Xu M, Lieberman I and Yang J: Finite Element Based-Analysis for Pre and Post Lumbar Fusion of Adult Degenerative Scoliosis Patients. Spine Deform 7: 543-552, 2019.

8. Zheng J, Yang Y, Cheng B and Cook D: Exploring the pathological role of intervertebral disc and facet joint in the development of degenerative scoliosis by biomechanical methods. Clin Biomech (Bristol, Avon) 70: 83-88, 2019.

9. Liu Y, Pan A, Hai Y, Li W, Yin L and Guo R: Asymmetric biomechanical characteristics of the paravertebral muscle in adolescent idiopathic scoliosis. Clin Biomech (Bristol, Avon) 65: 81-86, 2019.

10. Banno T, Arima H, Hasegawa T, Yamato Y, Togawa D, Yoshida G, Yasuda T, Oe S, Mihara Y, Ushirozako H, et al: The Effect of Paravertebral Muscle on the Maintenance of Upright Posture in Patients With Adult Spinal Deformity. Spine Deform 7: 125-131, 2019.

11. Dallaway A, Kite C, Griffen C, Duncan M, Tallis J, Renshaw D and Hattersley J: Age-related degeneration of the lumbar paravertebral muscles: Systematic review and three-level meta-regression. Exp Gerontol 133: 110856, 2020.

12. Crawford RJ, Volken T, Ni Mhuiris Á, Bow CC, Elliott JM, Hoggarth MA and Samartzis D: Geography of Lumbar Paravertebral Muscle Fatty Infiltration: The Influence of Demographics, Low Back Pain, and Disability. Spine 44: 1294-1302, 2019.

13. Ortega-Porcayo LA, Leal-López A, Soriano-López ME, Gutiérrez-Partida CF, Ramírez-Barrios LR, Soriano-Solis S, Rodríguez-García M, Soriano-Solis HA and Soriano-Sánchez JA: Assessment of Paraspinal Muscle Atrophy Percentage after Minimally Invasive Transforaminal Lumbar Interbody Fusion and Unilateral Instrumentation Using a Novel Contralateral Intact Muscle-Controlled Model. Asian Spine J 12: 256-262, 2018.

14. Kjaer P, Bendix T, Sorensen JS, Korsholm L and Leboeuf-Yde C: Are MRI-defined fat infiltrations in the multifidus muscles associated with low back pain? BMC Med 5: 2, 2007.

15. Kader DF, Wardlaw D and Smith FW: Correlation between the MRI changes in the lumbar multifidus muscles and leg pain. Clin Radiol 55: 145-149, 2000.

16. Hides JA, Stokes MJ, Saide M, Jull GA and Cooper DH: Evidence of lumbar multifidus muscle wasting ipsilateral to symptoms in patients with acute/subacute low back pain. Spine 19: 165-172, 1994.

17. Ranson CA, Burnett AF, Kerslake R, Batt ME and O'Sullivan PB: An investigation into the use of MR imaging to determine the functional cross sectional area of lumbar paraspinal muscles. Eur Spine J 15: 764-773, 2006.

18. Woodfield JC, Pettigrew RA, Plank LD, Landmann M and van Rij AM: Accuracy of the surgeons' clinical prediction of perioperative complications using a visual analog scale. World $\mathbf{J}$ Surg 31: 1912-1920, 2007.

19. Middendorp M, Vogl TJ, Kollias K, Kafchitsas K, Khan MF and Maataoui A: Association between intervertebral disc degeneration and the Oswestry Disability Index. J Back Musculoskeletal Rehabil 30: 819-823, 2017. 
20. Mmopelwa T, Ayhan S, Yuksel S, Nabiyev V, Niyazi A, Pellise F, Alanay A, Sanchez Perez Grueso FJ, Kleinstuck F, Obeid I, et al; European Spine Study Group (ESSG): Analysis of factors affecting baseline SF-36 Mental Component Summary in Adult Spinal Deformity and its impact on surgical outcomes. Acta Orthop Traumatol Turc 52: 179-184, 2018.

21. Xie D, Zhang J, Ding W, et al: Abnormal change of paravertebral muscle in adult degenerative scoliosis and its association with bony structural parameters. Eur Spine J 28: 1626-1637, 2019.

22. Schwab F, Dubey A, Gamez L, El Fegoun AB, Hwang K, Pagala M and Farcy JP: Adult scoliosis: Prevalence, SF-36, and nutritional parameters in an elderly volunteer population. Spine 30: 1082-1085, 2005.

23. Campbell PG and Nunley PD: The Challenge of the Lumbosacra Fractional Curve in the Setting of Adult Degenerative Scoliosis. Neurosurg Clin N Am 29: 467-474, 2018.

24. Simon MJK, Halm HFH and Quante M: Perioperative complications after surgical treatment in degenerative adult de novo scoliosis. BMC Musculoskelet Disord 19: 10, 2018.

25. Sun XY, Kong C, Lu SB, Wang W, Cheng YZ, Sun SY, Guo MC and Ding JZ: The Parallelogram Effect of Degenerative Structures Around the Apical Vertebra in Patients with Adult Degenerative Scoliosis: The Influence of Asymmetric Degeneration and Diagonal Degeneration on the Severity of Deformity. Med Sci Monit 25: 3435-3445, 2019.

26. Rustenburg CME, Kingma I, Holewijn RM, Faraj SSA, van der Veen A, Bisschop A, de Kleuver M and Emanuel KS Biomechanical properties in motion of lumbar spines with degenerative scoliosis. J Biomech: Nov 9, 2019 (Epub ahead of print).

27. Chon J, Kim HS, Lee JH, Yoo SD, Yun DH, Kim DH, Lee SA, Han YJ, Lee HS, Han YR, et al: Asymmetric Atrophy of Paraspinal Muscles in Patients With Chronic Unilateral Lumbar Radiculopathy. Ann Rehabil Med 41: 801-807, 2017.

28. Kang CH, Shin MJ, Kim SM, Lee SH and Lee CS: MRI of paraspinal muscles in lumbar degenerative kyphosis patients and control patients with chronic low back pain. Clin Radiol 62 : 479-486, 2007

29. Reza MM, Subramaniyam N, Sim CM, Ge X, Sathiakumar D, McFarlane C, Sharma $M$ and Kambadur R: Irisin is a pro-myogenic factor that induces skeletal muscle hypertrophy and rescues denervation-induced atrophy. Nat Commun 8: 1104, 2017.

30. Hiyama A, Katoh H, Sakai D, Tanaka M, Sato $M$ and Watanabe M: The correlation analysis between sagittal alignment and cross-sectional area of paraspinal muscle in patients with lumbar spinal stenosis and degenerative spondylolisthesis. BMC Musculoskelet Disord 20: 352,2019.

31. Hyun SJ, Bae CW, Lee SH and Rhim SC: Fatty Degeneration of the Paraspinal Muscle in Patients With Degenerative Lumbar Kyphosis: A New Evaluation Method of Quantitative Digital Analysis Using MRI and CT Scan. Clin Spine Surg 29: 441-447, 2016 .

32. Kalichman L, Carmeli E and Been E: The Association between Imaging Parameters of the Paraspinal Muscles, Spinal Degeneration, and Low Back Pain. BioMed Res Int 2017: 2562957, 2017

33. Fortin M, Gibbons LE, Videman T and Battié MC: Do variations in paraspinal muscle morphology and composition predict low back pain in men? Scand J Med Sci Sports 25: 880-887, 2015.

34. Zoabli G, Mathieu PA and Aubin CE: Back muscles biometry in adolescent idiopathic scoliosis. Spine J 7: 338-344, 2007.

35. Burakiewicz J, Sinclair CDJ, Fischer D, Walter GA, Kan HE and Hollingsworth KG: Quantifying fat replacement of muscle by quantitative MRI in muscular dystrophy. J Neurol 264 2053-2067, 2017.
36. Johnson BL, Schroeder ME, Wolfson T, Gamst AC, Hamilton G, Shiehmorteza M, Loomba R, Schwimmer JB, Reeder S, Middleton MS, et al: Effect of flip angle on the accuracy and repeatability of hepatic proton density fat fraction estimation by complex data-based, T1-independent, T $2 *$-corrected, spectrum-modeled MRI. J Magn Reson Imaging 39: 440-447, 2014.

37. Goubert D, Oosterwijck JV, Meeus M and Danneels L: Structural Changes of Lumbar Muscles in Non-specific Low Back Pain: A Systematic Review. Pain Physician 19: E985-E1000, 2016.

38. Wong E, Altaf F, Oh LJ and Gray RJ: Adult Degenerative Lumbar Scoliosis. Orthopedics 40: e930-e939, 2017.

39. Honkanen T, Mäntysaari M, Leino T, Avela J, Kerttula L, Haapamäki V and Kyröläinen H: Cross-sectional area of the paraspinal muscles and its association with muscle strength among fighter pilots: A 5-year follow-up. BMC Musculoskelet Disord 20: 170, 2019.

40. Xu M, Yang J, Lieberman I and Haddas R: Finite element method-based study for effect of adult degenerative scoliosis on the spinal vibration characteristics. Comput Biol Med 84: 53-58, 2017.

41. Wilke HJ, Wolf S, Claes LE, Arand M and Wiesend A: Stability increase of the lumbar spine with different muscle groups. A biomechanical in vitro study. Spine 20: 192-198, 1995.

42. Fortin M and Macedo LG: Multifidus and paraspinal muscle group cross-sectional areas of patients with low back pain and control patients: A systematic review with a focus on blinding. Phys Ther 93: 873-888, 2013.

43. Le Cara EC, Marcus RL, Dempsey AR, Hoffman MD and Hebert JJ: Morphology versus function: The relationship between lumbar multifidus intramuscular adipose tissue and muscle function among patients with low back pain. Arch Phys Med Rehabil 95: 1846-1852, 2014.

44. Hebert JJ, Le Cara EC, Koppenhaver SL, Hoffman MD, Marcus RL, Dempsey AR and Albert WJ: Predictors of clinical success with stabilization exercise are associated with lower levels of lumbar multifidus intramuscular adipose tissue in patients with low back pain. Disabil Rehabil 42: 679-684, 2020.

45. Glassman SD, Bridwell KH, Shaffrey CI, Edwards CC II, Lurie JD, Baldus CR and Carreon LY: Health-Related Quality of Life Scores Underestimate the Impact of Major Complications in Lumbar Degenerative Scoliosis Surgery. Spine Deform 6: 67-71, 2018.

46. Kim JB, Park SW, Lee YS, Nam TK, Park YS and Kim YB: The Effects of Spinopelvic Parameters and Paraspinal Muscle Degeneration on S1 Screw Loosening. J Korean Neurosurg Soc 58: 357-362, 2015.

47. Li H, Wang H, Zhu Y, Ding W and Wang Q: Incidence and risk factors of posterior cage migration following decompression and instrumented fusion for degenerative lumbar disorders. Medicine (Baltimore) 96: e7804, 2017.

48. Hyun SJ, Kim YJ and Rhim SC: Patients with proximal junctional kyphosis after stopping at thoracolumbar junction have lower muscularity, fatty degeneration at the thoracolumbar area. Spine J 16: 1095-1101, 2016.

(i) (5) This work is licensed under a Creative Commons Attribution-NonCommercial-NoDerivatives 4.0 International (CC BY-NC-ND 4.0) License. 